\title{
Vibration of Crankshaft-Propeller Systems. New Method of Calculation
}

\author{
M. A. BIOT \\ Columbia University
}

\section{ABSTRACT}

The calculation of torsional oscillations in crankshaft-propeller systems is carried out by a new method which reduces considerably the numerical work in the case of in-line engines. The theory is briefly outlined and the reader is referred to another publication of the author for further details. Three applications follow. In the first, all six natural frequencies and the corresponding modes are calculated for a V-12 engine. In the second example the method is adapted to the direct determination of the fundamental frequency. The third example deals with a 12-cylinder flat opposed engine coupled to a blower and through gears to a propeller; all eight natural frequencies are determined. The natural frequencies are determined by plotting a simple curve generally close to a straight line and the corresponding modes of oscillation in the crank are expressed in terms of a sine function. The amount of numerical work involved in the procedure is independent of the number of cylinders of the engine.

\section{INTRODUCTION}

$\mathbf{A}^{\mathrm{s}}$ REGARDS torsional oscillations, an internal combustion engine with a long crankshaft is generally considered to be equivalent to a uniform shaft carrying equidistant identical discs.

The procedures for deriving this equivalent system are familiar to vibration technicians. It is easy to calculate the dises; the moment of inertia of each is proportional to the average rotational inertia of each crank with the attached alternating masses. There will be as many dises as there are cranks. The calculation of the torsional rigidity of the equivalent shaft is not as straightforward. The crankshaft being a rather complicated elastic structure, it is generally difficult to evaluate exactly its average torsional rigidity. Moreover, it will depend on the bearing clearances. A practical rule is to adopt a shaft of the same length and diameter as the crankshaft, and, depending on one's judgment and experience, to vary this length slightly in accordance with bearing clearances, web rigidity, ${ }^{1}$ etc. The system is thus reduced to a shaft carrying a certain number of discs.

The various numerical methods devised to calculate the torsional oscillation of such a system become extremely tedious if the number of cranks exceed four. The object of the present paper is to show that it is possible to introduce considcrable simplification in this numerical work.

\section{THEORY}

Let $n$ be the number of discs representing the crankshaft, $I$ their moment of inertia, and $k$ the torsional

Received December 4, 1939. spring constant between these discs. Number the discs from 1 to $n$, and call $\theta_{x}$ the amplitude of oscillation of the disc numbered $x$ (Fig. 1). The amplitudes of oscillation of three successive dises satisfy the equation

$$
\theta_{x+1}-\left(2-\frac{I}{k} \omega^{2}\right) \theta_{x}+\theta_{x-1}=0
$$

where $\omega / 2 \pi$ is the frequency of the oscillation.

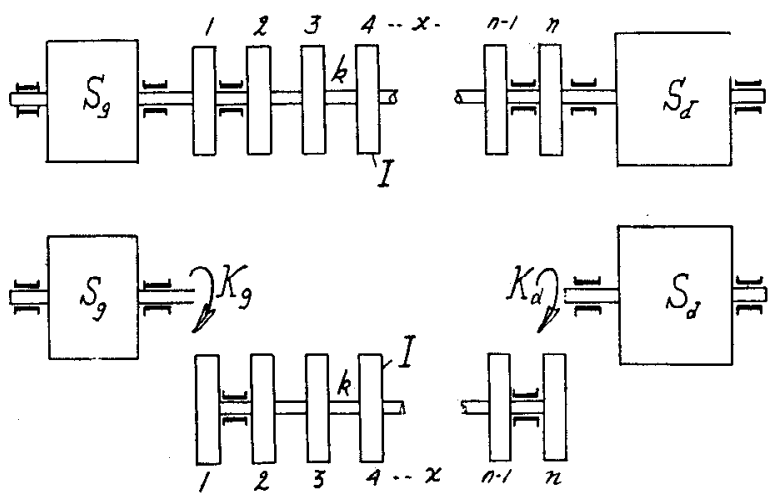

FIG. 1. Schematic representation of a crankshaft and its end impedances.

Setting

$$
\omega=2 \sqrt{k / I} \sin \mu / 2
$$

it can be verified that Eq. (1) is satisfied by the solution

$$
\theta_{x}=A \cos \mu x+B \sin \mu x
$$

in which $A$ and $B$ are arbitrary constants. These arbitrary constants are determined by the two relations

$$
\begin{array}{r}
\theta_{2}-\left(1-\frac{I}{k} \omega^{2}-K_{g} / k\right) \theta_{1}=0 \\
\theta_{n-1}-\left(1-\frac{I}{k} \omega^{2}-K_{d} / k\right) \theta_{n}=0
\end{array}
$$

which govern the motion of the dises at the ends of the shaft. Eqs. (4) involve the mechanical impedances $K_{g}$ and $K_{d}$ (Fig. 1) of those parts of the engine which are coupled to the discs number 1 and number $n$, respectively. The method for calculating these impedances, which are in general functions of the frequency, will be shown in connection with the numerical examples below.

The substitution of the general solution (3) into Eqs. (4) leads to the conditions 
$A\left[1+\left(\frac{K_{\varepsilon}}{k}-1\right) \cos \mu\right]+B\left(\frac{K_{g}}{k}-1\right) \sin \mu=0$

$A\left[\cos \mu(n+1)+\left(\frac{K_{d}}{k}-1\right) \cos \mu n\right]+$

$$
B\left[\sin \mu(n+1)+\left(\frac{K_{d}}{k}-1\right) \sin \mu n\right]=0
$$

By elimination of $A$ and $B$ one obtains the frequency equation,

$$
\begin{aligned}
& \sin \mu(n+1)+\left(\frac{K_{g}+K_{d}}{k}-2\right) \sin \mu n+ \\
& \left(\frac{K_{g}}{k}-1\right)\left(\frac{K_{d}}{k}-1\right) \sin \mu(n-1)=0
\end{aligned}
$$

This equation contains the unknown frequency $\omega / 2 \pi$ in the variable $\mu$ and in the end impedances $K_{g}$ and $K_{d}$. The number of cranks $n$ enters as a parameter. The reader will find a more detailed derivation of Eq. (6) and further information on mechanical impedances in reference 2 .

In principle, in order to find the roots of the frequency equation (6) it would be sufficient to plot the left side of the equation as a function of $\mu$ or $\omega$ and note the value of the abscissa where the curve intersects the horizontal axis. This procedure, however, is rather cumbersome because the function to be plotted goes through many oscillations and requires the calculation of a great number of points. This difficulty is avoided by introducing the complex quantities

$$
\begin{aligned}
& e^{\frac{\mu i}{2}}+\left(\frac{K_{g}}{k}-1\right) e^{-\frac{\mu i}{2}}=A_{g} e^{\phi g^{i}} \\
& e^{\frac{\mu i}{2}}+\left(\frac{K_{d}}{k}-1\right) e^{-\frac{\mu i}{2}}=A_{d} e^{\phi_{d i}}
\end{aligned}
$$

The left side of Eq. (6) is then the imaginary part of $A_{g} A_{d} e^{\left(\mu n+\phi_{g}+\phi_{d}\right) i}$. An equivalent form of the frequency equation (6) is therefore

$$
\mu n+\phi_{g}+\phi_{d}=\text { multiple of } \pi
$$

The quantities $\phi_{g}$ and $\phi_{d}$ are functions of $\mu$ or $\omega$ defined by Eqs. (7). Their values are

$$
\begin{aligned}
& \phi_{g}=\tan ^{-1}\left[\left(\frac{2 k}{K_{g}}-1\right) \tan \frac{\mu}{2}\right] \\
& \phi_{d}=\tan ^{-1}\left[\left(\frac{2 k}{K_{d}}-1\right) \tan \frac{\mu}{2}\right]
\end{aligned}
$$

The practical advantage of Eq. (8) over Eq. (6) resides in the fact that the left side $\mu n+\phi_{g}+\phi_{d}$ plotted as a function of $\mu$ is a curve generally near to a straight line. The form (8) of the frequency equation is therefore well fitted for a solution by graphical methods or interpolation. It is sufficient to plot the curve in the range between $\mu=0$ and $\mu=180^{\circ}$.

It is also of importance to the designer to know not only the frequencies of the natural oscillations, but to estimate their respective danger as regards resonance stresses. This is done by calculating the energy input of the pressure cycles in each mode. The energy input depends on the Fourier harmonics of the pressure cycle, the firing order of the engine and the shape of the torsional modes in the crankshaft. These shapes are easily obtained from the general solution, Eq. (3) and conditions (5). The angular amplitude of the crank numbered $x$ is

$$
\theta_{x}=C \sin (\mu x+\beta)
$$

where $\beta$ is defined by the relation

$$
\tan \beta=\frac{\left(1-K_{g} / k\right) \sin \mu}{1+\left(K_{g} / k-1\right) \cos \mu}
$$

The constant $C$ is arbitrary. The torsional mode of order $r$ is found by substituting in these formulas the values $\mu_{2}$ and $\omega_{r}$ corresponding to that mode.

\section{APPLICATIONS}

\section{Example 1}

A V-12 engine is represented schematically in Fig. 2. It is coupled directly to a propeller through a shaft of spring constant $k_{1}$. The moment of inertia of the propeller is $I_{1}$. The numerical values are

$$
\begin{aligned}
I & =.415 \mathrm{lb} . \text { in. sec. } \\
I_{1} & =162 \mathrm{lb} . \text { in. sec. } \\
k & =5.10 \text { times } 10^{6} \mathrm{lb} . \text { in. per rad. } \\
k_{1} & =2.05 \text { times } 10^{6} \mathrm{lb} . \text { in. per rad. } \\
n & =6
\end{aligned}
$$

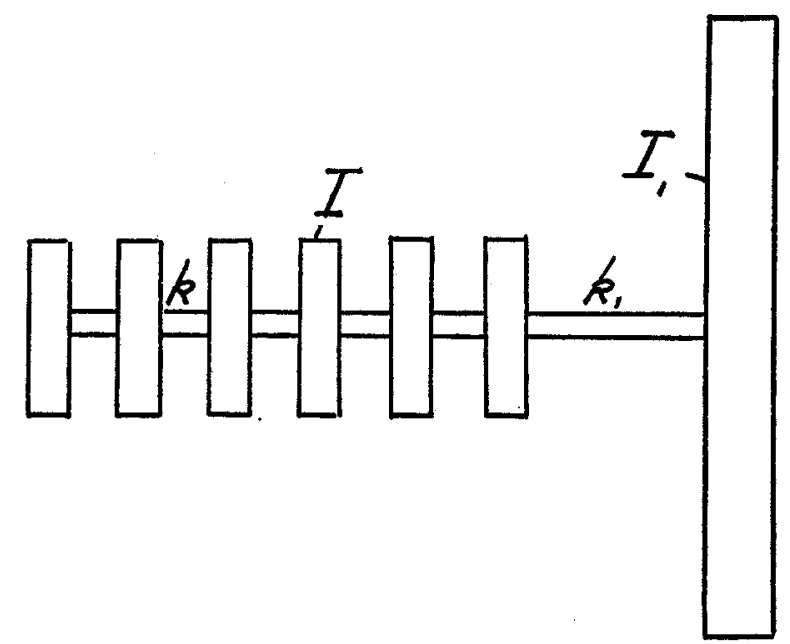

Fig. 2. V-12 engine with propeller.

The moment of inertia $I_{1}$ being very large compared to $6 I$, it is assumed that the propeller does not oscillate.* The mechanical impedance on the propeller side is therefore reduced to $K_{d}=k$, i.e., it is equal to the spring constant of the propeller shaft itself. From Eq. (9),

* In all examples treated here the propeller is assumed to be rigid. The influence of propeller elasticity will be taken up in a subsequent paper. 


$$
\phi_{d}=\tan ^{-1}\left[\left(\frac{2 k}{k_{1}}-1\right) \tan \frac{\mu}{2}\right]
$$

On the left the crankshaft is free so that $K_{\mathrm{g}}=0$; hence $\phi_{g}=90^{\circ}$ is a constant. Expressing all angles in degrees,

$\begin{array}{rcc}\mu & \phi_{d} & 6 \mu+\phi_{o}+\phi_{d} \\ 0 & 0 & 90 \\ 15 & 27.2 & 207.2 \\ 30 & 46.2 & 316.2 \\ 45 & 58.2 & 418.2 \\ 60 & 66.0 & 516.0 \\ 75 & 71.3 & 611.3 \\ 90 & 75.6 & 705.8 \\ 120 & 81.6 & 891.6 \\ 150 & 86.0 & 1076.0 \\ 180 & 90.0 & 1260.0\end{array}$

The values $6 \mu+\phi_{g}+\phi_{d}$ are plotted as functions of $\mu$ in Fig. 3. The intersections of this curve with

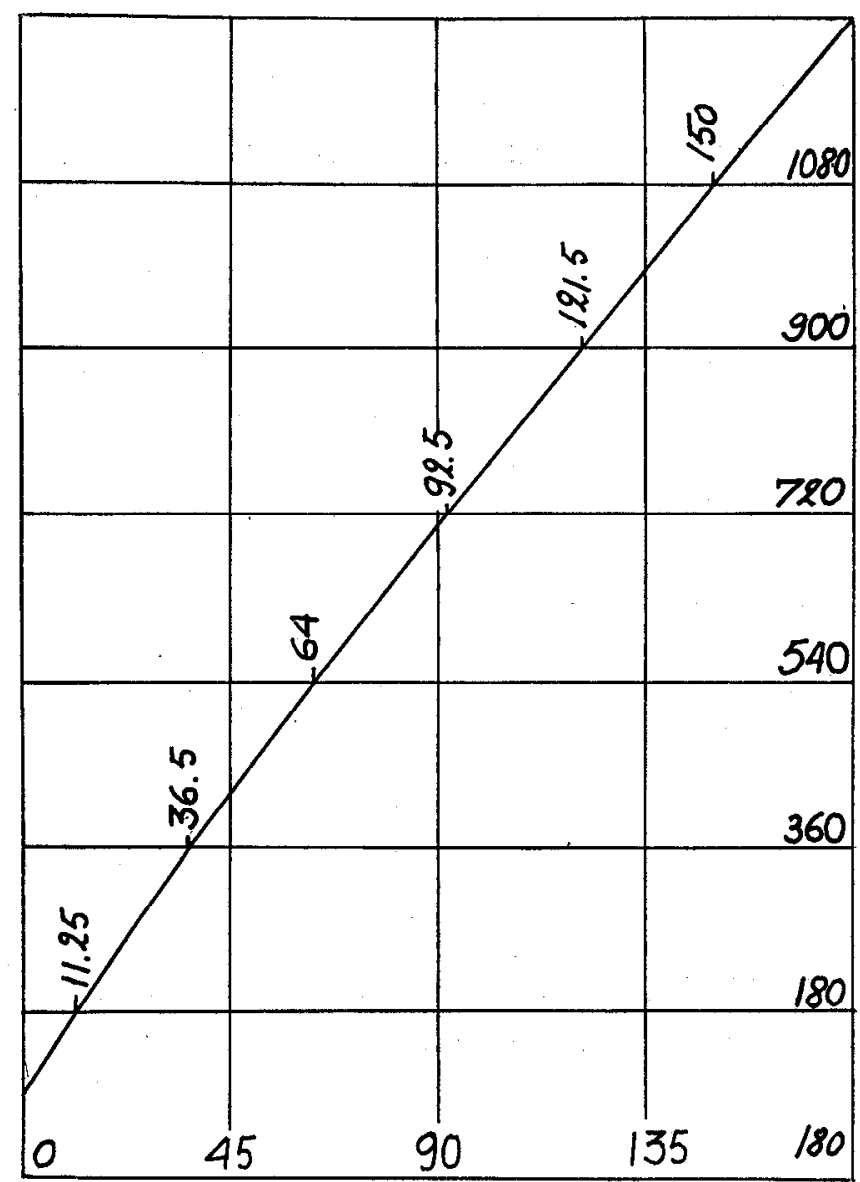

FIG. 3. Graphical determination of the natural frequencies of the engine in Fig. 2 by plotting $6 \mu+\phi_{g}+\phi_{d}$ as a function of $\mu$.

the horizontals of ordinates $180^{\circ}, 2 \times 180^{\circ}, 3 \times 180^{\circ}$, ... etc., yield six roots of the frequency equation (8). These roots are the abscissas of the points of intersection and their values in degrees are

$$
\begin{array}{lll}
\mu_{1}=11.25 & \mu_{2}=36.5 & u_{3}=64 \\
\mu_{4}=92.5 & \mu_{5}=121.5 & \mu_{6}=150
\end{array}
$$

The corresponding natural frequencies are derived from Eq. (2). In cycles per minute,

$$
f_{r}=\frac{60}{\pi} \sqrt{\frac{k}{I}} \sin \frac{\mu_{r}}{2}
$$

$f_{1}=66800 \sin 5.64=6560$ per $\min$.

$f_{2}=66800 \sin 18.25=20,900$ per min.

$f_{3}=66800 \sin 32.0=35,400$ per $\min$.

$f_{4}=66800 \sin 46.2=48,200$ per min.

$f_{5}=66800 \sin 60.7=58,300$ per min.

$f_{6}=66800 \sin 75.0=64,500$ per $\min$.

From Eqs. (10) and (11) the shape of the torsional modes are derived. Since $K_{g}=0, \tan \beta=\sin \mu /$ $(1-\cos \mu)=1 / \tan (\mu / 2)$; hence $\beta=(\pi-\mu) / 2$. The shape of the $r^{\text {th }}$ mode is therefore

\begin{tabular}{|c|c|c|c|c|c|c|}
\hline \multicolumn{2}{|r|}{ 1st Mode } & nd Mode & rd Mode & th Mod & th Mode & th Mode \\
\hline$\theta_{1}$ & .995 & .949 & .848 & .692 & .489 & .258 \\
\hline$\theta_{2}$ & .956 & .576 & -.104 & -.743 & -.999 & -.707 \\
\hline$\theta_{8}$ & .881 & -.024 & -.939 & -.642 & .544 & .965 \\
\hline$\theta_{4}$ & .785 & -.615 & -.719 & .798 & .438 & -.961 \\
\hline$\theta_{3}$ & .633 & -.961 & .309 & .573 & -.994 & .694 \\
\hline$\theta_{6}$ & .469 & -.933 & .990 & -.838 & .601 & -.258 \\
\hline
\end{tabular}

$$
\theta_{x}=\cos \mu_{r}(x-1 / 2) ; \quad x=1,2, \ldots .6
$$

The shape of all six modes is given in the following table and represented in Fig. 4.
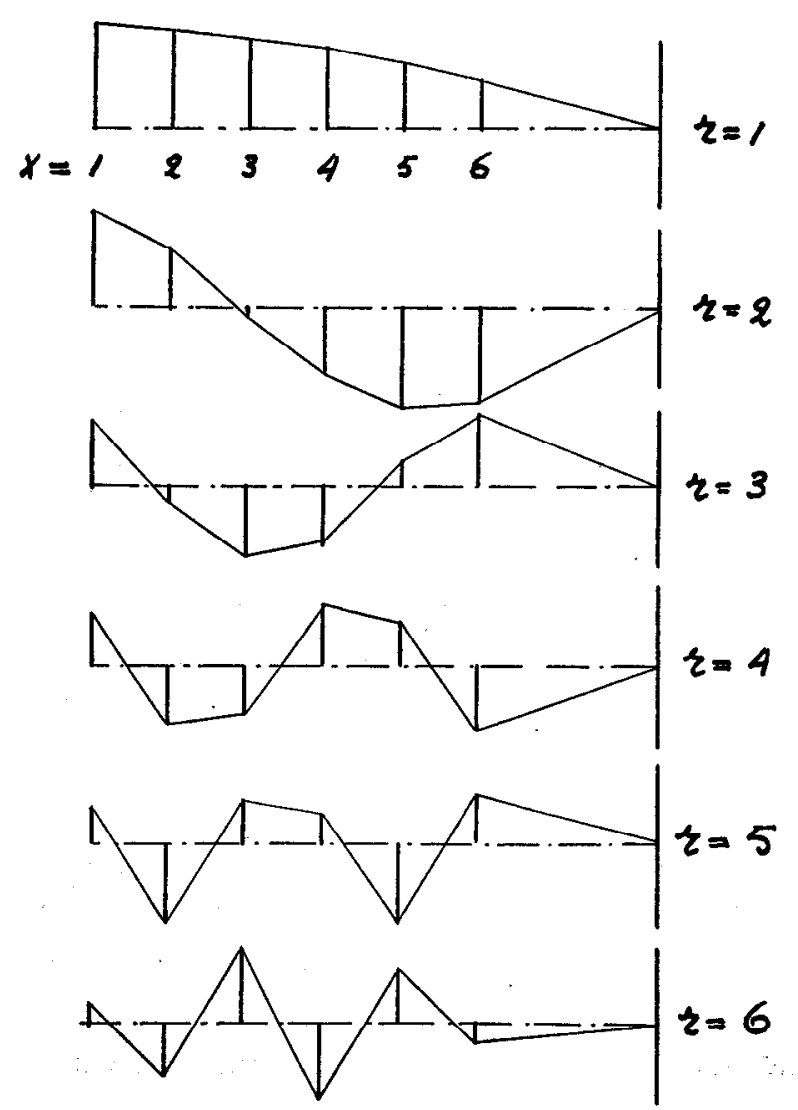

Frg. 4. The six torsional modes of the engine in Fig. 2. 
It will be noticed that the problem is simplified if the spring constant of the propeller shaft is equal to $k$. Putting $k_{1}=k$,

$$
\phi_{d}=\tan ^{-1}[\tan (\mu / 2)]=\mu / 2
$$

and the expression $6 \mu+\phi_{d}+\phi_{g}=6 \mu+1 / 2 \mu+90^{\circ}$ plotted as a function of $\mu$ comes out as a straight line In this case the roots $\mu_{r}$ are

$$
\mu_{r}=(2 / 13)(r-1 / 2) \pi ; \quad r=1,2, \ldots \ldots 6
$$

\section{Example II}

Consider the same engine and propeller as in the previous case but with a drive shaft of low rigidity (Fig. 5). The numerical values are

$$
\begin{aligned}
I & =.415 \mathrm{lb} . \text { in. } \text { sec. }^{2} \\
I_{1} & =162 \mathrm{lb} . \text { in. } \text { sec. }^{2} \\
k & =5.10 \text { times } 10^{6} \mathrm{lb} . \text { in. per rad. } \\
k_{1} & =.46 \text { times } 10^{6} \mathrm{lb} . \text { in. per rad. } \\
n & =6
\end{aligned}
$$

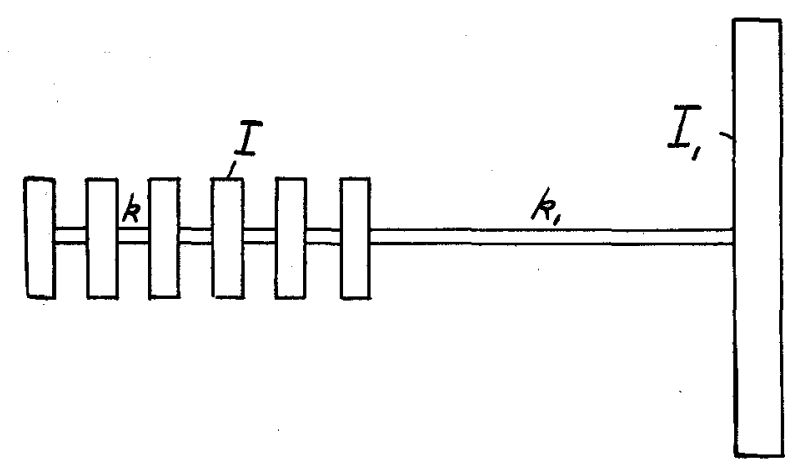

FIG. 5. V-12 engine with propeller and extension drive shaft.

One may be interested primarily in the fundamental frequency which in this case is low compared to the harmonics. It is possible to take advantage of this fact to calculate the fundamental mode directly in the following way.

Putting $K_{g}=0, K_{d}=k_{1}$, Eq. (6) is put in the form

$$
\lambda I \omega^{2}=k_{1}
$$

with

$$
\lambda=\frac{\sin \mu n}{2 \sin (\mu / 2) \cos \mu(n-1 / 2)}
$$

For small values of $\mu, \lambda$ is approximately equal to $n$. Hence in this case,

$$
6 I \omega^{2}=k_{1} \text { (approximately) }
$$

This equation could have been obtained directly by assuming the crankshaft to behave as a rigid body.

$$
\omega_{1}=\sqrt{\frac{k_{1}}{6 I}}=428
$$

and from Eq. (2) the corresponding value of $\mu$ is

$$
\mu_{1}=7.0^{\circ}
$$

Substituting this value of $\mu_{1}$ in $\lambda$,

$$
\lambda=7.02
$$

and therefore a second approximation is

$$
\omega_{1}=\sqrt{k_{1} / 7.02 I}=397 ; \mu_{1}=6.5^{\circ}
$$

This second approximation is quite satisfactory. The fundamental frequency is

$$
f_{1}=30 \omega_{1} / \pi=3800 \text { per min. }
$$

The shape of the fundamental mode is derived from Eq. (10), in which $\mu_{1}=6.5^{\circ}$ is substituted, and $\beta=$ $(\pi-\mu) / 2$. This mode is represented in Fig. 6 .

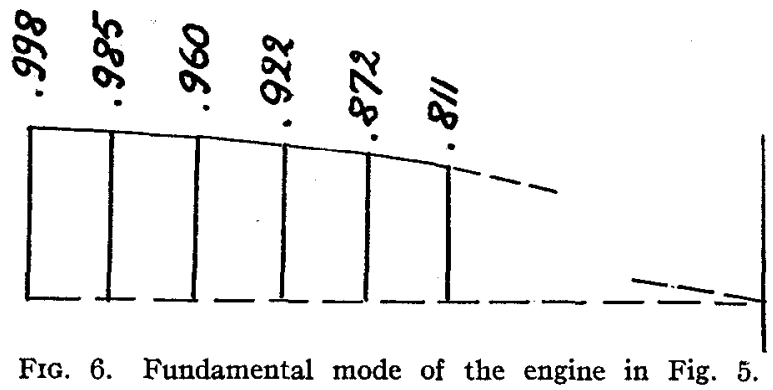

\section{Example III}

Consider a 12-cylinder flat opposed engine with propeller, reduction gear, and blower. The system is represented schematically in Fig. 7. The numerical values corrected to crankshaft speed are as follows*:

$$
\begin{aligned}
I & =.65 \mathrm{lb} . \text { in. sec. } \\
I_{1} & =.49 \mathrm{lb} . \text { in. sec. } \\
I_{2} & =155 \mathrm{lb} . \text { in. sec. } \\
I_{3} & =5.95 \mathrm{lb} . \text { in. sec. }^{2} \\
k & =10.5 \text { times } 10^{6} \mathrm{in.} \text { lb. per rad. } \\
k_{1} & =8 \text { times } 10^{6} \mathrm{in} . \mathrm{lb} . \text { per rad. } \\
k_{2} & =.6 \text { times } 10^{6} \text { in. } 1 \mathrm{~b} . \text { per rad. } \\
k_{3} & =.05 \text { times } 10^{6} \mathrm{in.} \text { lb. per rad. } \\
n & =6
\end{aligned}
$$

The reciprocal of the mechanical impedance $K_{d}$ corresponding to the propeller and gears may be calculated as a function of $\mu$ through the following steps

* The author is indebted for the data on this engine to Mr. L. S. Hobbs and Mr. Williams of Pratt and Whitney Aircraft.

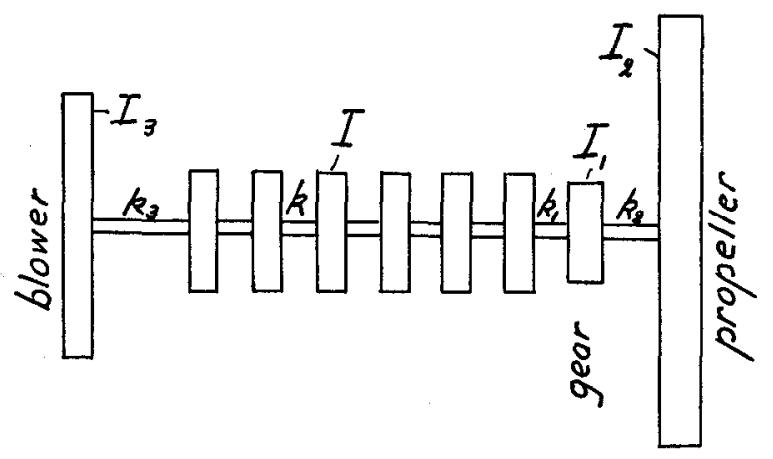

Frg. 7. 12 cylinder flat opposed engine with gear propeller and blower. 


$$
\begin{aligned}
\omega^{2} & =\frac{4 k}{I} \sin ^{2} \frac{\mu}{2} \\
\frac{1}{K_{d}^{\prime \prime}} & =\frac{1}{k_{2}}-\frac{1}{I_{2} \omega^{2}} \\
K_{d}^{\prime} & =K_{d}^{\prime \prime}-I_{1} \omega^{2} \\
\frac{1}{K_{d}} & =\frac{1}{k_{1}}+\frac{1}{K_{d}^{\prime}}
\end{aligned}
$$

The impedance on the blower side is given by

$$
\frac{1}{K_{g}}=\frac{1}{k_{3}}-\frac{1}{I_{3} \omega^{2}}
$$

The corresponding functions $\phi_{g}$ and $\phi_{d}$ defined by Eqs. (9) are then calculated.

Before doing this, however, it is convenient to take advantage of the fact that approximate values for the two lower frequencies are easily found. The fundamental frequency corresponds to an oscillation of the blower while the engine and propeller stay fixed. This gives

$$
\omega_{1}=\sqrt{k_{3} / I_{3}}=91.7 ; f_{1}=876 \text { per min. }
$$

and the corresponding value of $\mu$ is $\mu_{1}=1.30^{\circ}$

The next frequency corresponds to an oscillation of the mass $6 I+I_{1}$ as a rigid system while the propeller and the blower stay fixed. Due to the low valuc of $k_{3}$ the influence of the blower on this frequency is negligible. The second frequency is therefore approximately

$$
\omega_{2}=\sqrt{\frac{k_{2}}{6 I+I_{1}}}=370 ; f_{2}=3530 \text { per min. }
$$

and the corresponding value of $\mu$ is

$$
\mu_{2}=5.30^{\circ}
$$

Having obtained approximate values for the two lower roots the calculation of $\phi_{g}$ and $\phi_{d}$ in the range $0<\mu$ $<15^{\circ}$ is limited to three points in the vicinity of each value $\mu_{1}$ and $\mu_{2}$. The functions are given in the following table.

\begin{tabular}{rccc}
$\mu$ & $\phi_{g}$ & $\phi_{d}$ & $6 \mu+\phi_{g}+\phi_{d}$ \\
0 & -90 & -90 & -180 \\
1.25 & -25.5 & 11.75 & -6.3 \\
1.30 & -7.1 & 12.9 & 13.6 \\
1.35 & 13.8 & 14.1 & 36.0 \\
4 & 85.6 & 52.2 & 161.8 \\
5 & 86.6 & 59.5 & 176.1 \\
6 & 87.2 & 66.3 & 189.5 \\
15 & 88.9 & 88.7 & 267 \\
30 & 89.5 & 107 & 376 \\
45 & 90 & 124 & 484 \\
60 & 90 & 144 & 594 \\
75 & 90 & 169 & 709 \\
90 & 90 & 194 & 824 \\
120 & 90 & 231 & 1041 \\
150 & 90 & 253 & 1243 \\
180 & 90 & 270 & 1440 \\
\hline
\end{tabular}

The quantity $6 \mu+\phi_{g}+\phi_{d}$ is plotted as function of $\mu$ in Fig. 8. The roots determined graphically are

$$
\begin{array}{llll}
\mu_{1}=1.268 & \mu_{2}=5.30 & \mu_{3}=27.8 & \mu_{4}=53 \\
\mu_{5}=77 & \mu_{6}=101 & \mu_{7}=126 & \mu_{8}=152
\end{array}
$$

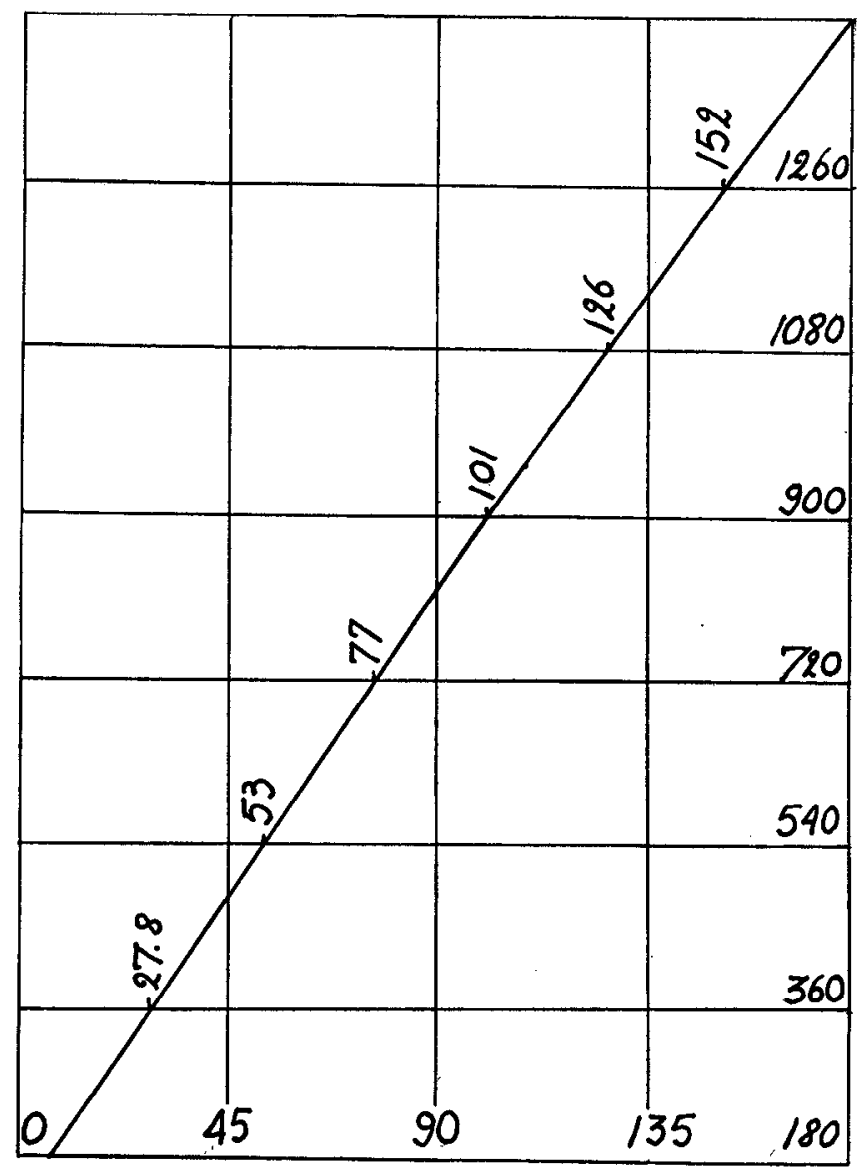

FIG. 8. Graphical determination of the natural frequencies of the engine in Fig. 8 by plotting $6 \mu+\phi_{\sigma}+\phi_{d}$ as a function of $\mu$. The curve is not plotted in the range $0<\mu<6^{\circ}$ and the two lower roots $\mu_{1}$ and $\mu_{2}$ do not appear in the diagram.

The corresponding frequencies in cycles per min. are:

$$
\begin{aligned}
& f_{1}=76800 \sin .634=846 \text { per min. } \\
& f_{2}=76800 \sin 2.65=3540 \text { per min. } \\
& f_{3}=76800 \sin 13.9=18500 \text { per min. } \\
& f_{4}=76800 \sin 26.5=34200 \text { per min. } \\
& f_{5}=76800 \sin 38.5=47700 \text { per min. } \\
& f_{6}=76800 \sin 50.5=59200 \text { per min. } \\
& f_{7}=76800 \sin 63 \quad=68400 \text { per min. } \\
& f_{8}=76800 \sin 76 \quad=74500 \text { per min. }
\end{aligned}
$$

A complete plot of the curve $6 \mu+\phi_{g}+\phi_{d}$ would show that it is not as near to a straight line in the range $0<\mu<6^{\circ}$. However in the range $1.25^{\circ}<\mu<1.35^{\circ}$ and $4^{\circ}<\mu<6^{\circ}$ it is practically straight so that $\mu_{1}$ and $\mu_{2}$ may be determined quite accurately by linear interpolation.

\section{Conclusion}

A simple expression (Eq. (6)) has been developed for the natural frequencies of torsional oscillation of a 
crankshaft-propeller system. In this equation the number of cranks appears as a parameter. A further simplification resides in the possibility of determining the roots of this equation graphically by plotting a curve (Eq. (8)) which is near to a straight line and therefore requires the calculation of only a few points. Once the frequencies are found it is easy to determine the energy input in each mode since the shape of each mode in the crank is expressed by means of a simple sine function (Eq. (10)). An idea of the rapidity of the method is given by the fact that the calculation of the six natural frequencies and their corresponding modes in Example I takes about one and a half hours of slide rule work. This is considerably faster than by any other method. Other advantages are: the necessary smoothness of the plotted curve furnishes an immediate check on any numerical error; the amount of numerical work is independent of the number of cylinders; possibility of calculating the new frequencies due to a separate change in propeller, crankshaft, or blower, without having to repeat all of the computations; possibility of taking advantage of an approximate guess of certain frequencies. The method is also applicable as such to engines with double identical crankshafts in parallel.

\section{REFERENCES}

1 Den Hartog, J. P., Mechanical Vibrations, page 205; McGrawHill Book Co., 1934.

2 v. Kármán, Th., and Biot, M. A., Mathematical Methods in Engineering, Chapter XI, Section 6; McGraw-Hill Book Co., 1940.

${ }^{3}$ Lürenbaum, Karl, Vibration of Crankshaft-Propeller Systems, S.A.E. Journal, Vol. 39, pages 469-479, December, 1936. 